\title{
Photovoltaic power maximum power point tracking technology based on the fractional-step perturbation observation method
}

\author{
Xu Shu-wei ${ }^{1} \&$ Dong Yan ${ }^{1} \&$ Zhang Yiyang ${ }^{1}$ \\ Li Lian-feng ${ }^{2} \&$ Zeng Jing-yu² \& Cai Hong-wang ${ }^{2}$
}

${ }^{1}$ ChangchunInstitute of Engineering Technology Department of electronic engineering 130117

${ }^{2}$ ChangChun University of Technology Department of electronic engineering 130012

KEYWORD: Solar Power; Maximumpower point ; Track; Perturbation and observation method ABSTRACT: In order to obtain the maximum power output of the solar cell, the output power should be continuously monitored in real time. Based on the traditional perturbation and observation methods, we propose a new algorithm of maximum power point tracking. The method uses the slope of the P-V curve to determine the disturbance step, which improves the tracking performance. Simulation results show that this method is superior performance.

\section{PHOTOVOLTAIC POWER MAXIMUM POWER POINT (MPPT) TRACKING}

In order to improve the power generation efficiency of the photovoltaic power generation system and reduce production costs, from the view of electronic systems, the MPPT (Maximum Power Point Tracking, referred MPPT) is a preferred solution because it is simpler, faster and more effective.

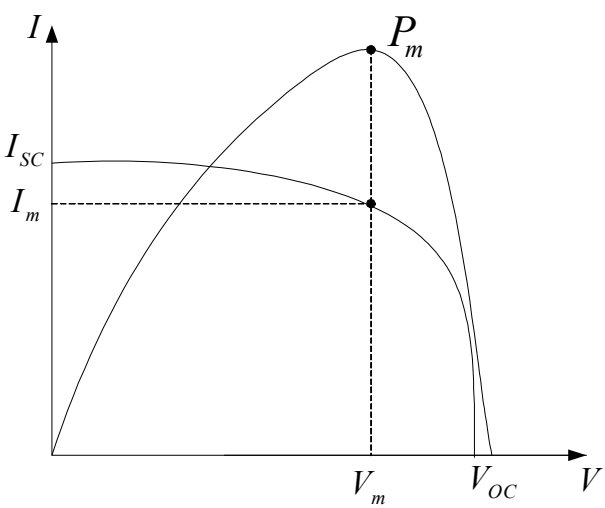

Figure 1 shows a characteristic curve of the solar cell, which I-V characteristic curve with a solid line to present, P-V characteristic curve with dotted lines showing.

Figure1. Solar cells characteristic curve

Among them,

$I_{S C}$ : Short circuit current, which is the biggest current of solar cell output under specific temperature and sunshine intensity;

$I_{m}$ : Current of maximum power point, which refers to the current of maximum power output of solar cell under a specific temperature and sunlight intensity;

$V_{O C}:$ Open circuit voltage, which refers to the voltage of maximum output of solar cell under a specific temperature and sunshine intensity;

$V_{m}$ : Maximum power point voltage, which is to point to voltage value of maximum power output of solar cell under a specific temperature and sunlight intensity.

It can be clearly seen on the solar cell IV characteristic curve that at a particular light intensity and relative humidity conditions, the maximum power output point $P_{m}$ can always be found on the so- 
lar cell output curve, so better methods can be found to solve this problem. In order to get the maximum power output of the solar cell, the output power of solar cell should be continuously monitored in real time. In actual operation, the operating point of the PV array needs to be adjusted to make it work in the vicinity of the maximum power point, which is called optimization.

It is well-known that there are many ways to calculate the maximum power point tracking ${ }^{[1-3]}$, such as constant tracking method, the incremental conductance method, perturbation and observation method and batch scanning. Characteristics of constant voltage tracking method is relatively higher reliability, simpler to operate and easier to control, and the entire power system is less prone to significant change in the oscillation. In other words, this method improves the stability of the system. But the disadvantage is also obvious, such as the accuracy of control range is not high, and dramatic changes in sunlight intensity and temperature difference between the relatively large area is not applicable. Therefore, this algorithm is being gradually replaced by other algorithms. Incremental conductance method and perturbation and observation method are substantially the same principle. The most obvious difference is that the former uses predicate logic, the latter is for certain measurement parameters necessary trade-offs. Implement incremental conductance method is relatively complex, in terms of temperature and speed detection, the method affect the tracking power point, which increase the difficulty of control and calculation ${ }^{[4]}$.

\section{PERTURBATION AND OBSERVATION METHOD}

Fixed step disturbance observation method is based on the first output current in operation of the PV array for accurate measurements, and secondly in the original working state of the system, to artificially increase the output voltage of a voltage component (or called disturbance), eventually the change of output power system can be detected, and we will use the change of power varying sizes to find the maximum power point of photovoltaic power generation system. If the relationship between a given output power voltage and the system actual voltage was proportional which proves the location of the maximum power point is on the right of the current operating point, the next step is to further increase the voltage; if the relationship was inversely, which proves the left position of the maximum power point in the current operating point, we need to further reduce the voltage. Finally, the desired effect is achieved at the maximum power point working point near the work area.

The advantage of perturbation and observation method is that it is reliable and simple to operate, relatively easier to implement. The disadvantage is that it is not easy to define the step, in other words, if the step is too short or too long, the tracking performance of the system will be directly affected. It is not sure to determine the position of the maximum power point. Changes in the external environment is also likely to lead to misjudgment of the system, For example, when the system is working $25^{\circ} \mathrm{C}$ of ambient temperature, the radiation intensity of light is $800 \mathrm{~W} / \mathrm{m}^{2}$, this time looking for the maximum power point, the measured direction to decrease the disturbance. When the system is still working $25^{\circ} \mathrm{C}$ of outside ambient temperature, when the light radiation intensity becomes $1000 \mathrm{~W} / \mathrm{m}^{2}$, after the increase in the disturbance, estimates the system power increases, so we could easily determine the direction of the disturbance is still decreasing, but the actual disturbance voltage should be increased, this is the system in the external environment changes, made the wrong judgment.

Calculate flow chart is shown in Figure 2, in which Vold and Iold, respectively, the value of the first $k-1$ second solar cell output voltage and current ${ }^{[5]}$. 


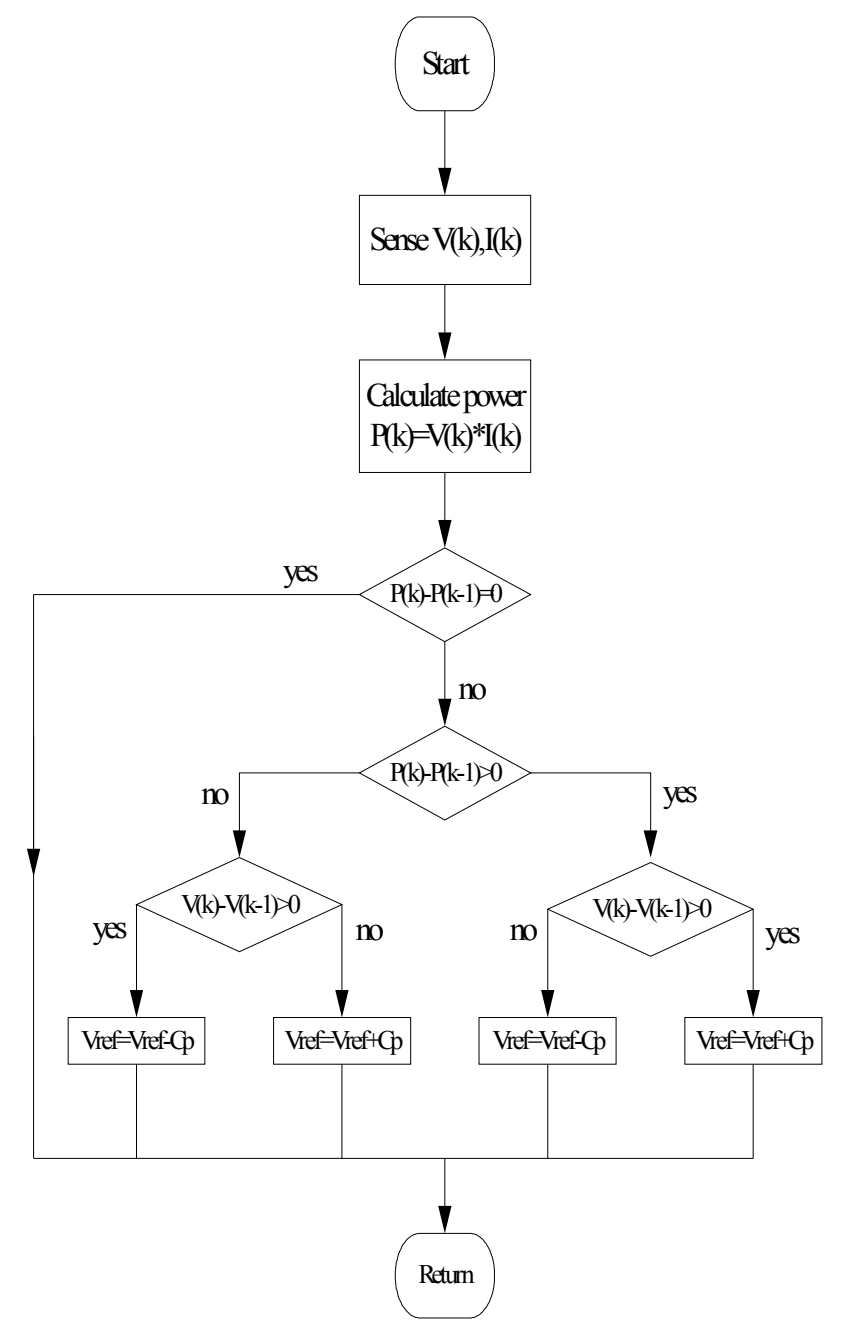

Figure 2. The flow chart of disturbance observation

\section{FRACTIONAL STEP PERTURBATION AND OBSERVATION METHOD BASED ON P-V CURVE SLOPE}

Based on the perturbation and observation method, we proposed fractional step perturbation and observation based on P-V curve slope.

Through solar cell P-V characteristic curve (Figure 1), it can be known that the slope of the P-V curve at the maximum power point is zero. According to the knowledge of advanced mathematics, when the points on the left side of the curve are close to the maximum point, the slope of the curve is smaller and closer to zero. In the same way, the absolute value of the slope of the curve is smaller and closer to zero. Therefore, the perturbation step size and P-V curve slope and can be used to establish a relationship between the two, namely when in the P-V curve of slope absolute value smaller, perturbation step should be decreased, whereas it should be increased.

The algorithm flow chart of the slope of the P-V curve is based on the fractional step method. The flow chart is shown in Figure 2: P (0) is the previous output power of system, DV is the variation of previous voltage, $\mathrm{V}$ and I respectively represent the solar cell voltage and the output current value, $\mathrm{P}$ (k) for measurement the system output power, the temp to a temporary variable, Vref is the voltage value to be output from a photovoltaic cells next time. 


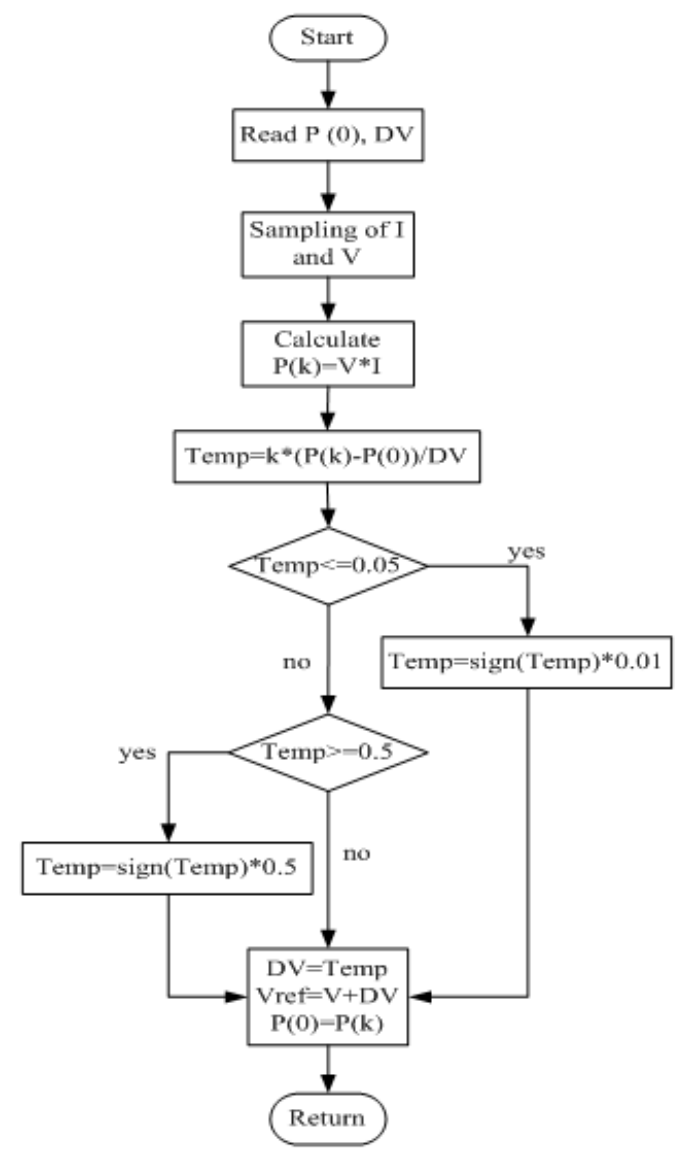

Figure 3. Flow chart of the step by step method based on the slope of P-V curve

In Figure 3, formula Temp=k*( $\mathrm{P}(\mathrm{k})-\mathrm{P}(0)) / \mathrm{DV}$ is the core of this method, by which can be known that the perturbation step is determined by the slope of the P-V curve. The ratio of $\mathrm{P}(\mathrm{k})-\mathrm{P}(0)$ with DV can be very good to determine the positive and negative step, so as to achieve the purpose of improving the efficiency of the whole system. For the proportion coefficient k, usually take 1. Any situation can occur in real working environment. When temperature changes more frequently and light intensity changes obviously, large disturbance step will also change, so it is necessary to control its step length variation to achieve the optimal control results. Through a series of simulation research, we determine the value of 0.5 , that is the value of step length variation smaller than 0.5 is optimal, and it needs to be forced to 0.5 if greater.

When the whole system is in static mode, the operation of the system over a period of time tracking, perturbation step size becomes zero, with the power becomes zero, at this time there will be Temp = $\mathrm{k} *(\mathrm{P}(\mathrm{k})-\mathrm{P}(0)) / \mathrm{DV}$ the denominator is zero, it is not reasonable, once the external environment changes, and the whole system has lost the ability to continue to follow, so as long as when disturbance step length is less than 0.05 , forced to as 0.01 , in order to achieve the purpose of improve the static characteristic of the algorithm.

Several parameters mentioned above are determined by calculation and simulation. In actual working environment, they can be determined according to actual situation.

\section{SIMULATION RESULTS}

\section{The solar cell Simulation Model}

Use function module to establish the internal structure of solar cell simulation model shown in Figure4 in Simulink environment of MATLAB software. 


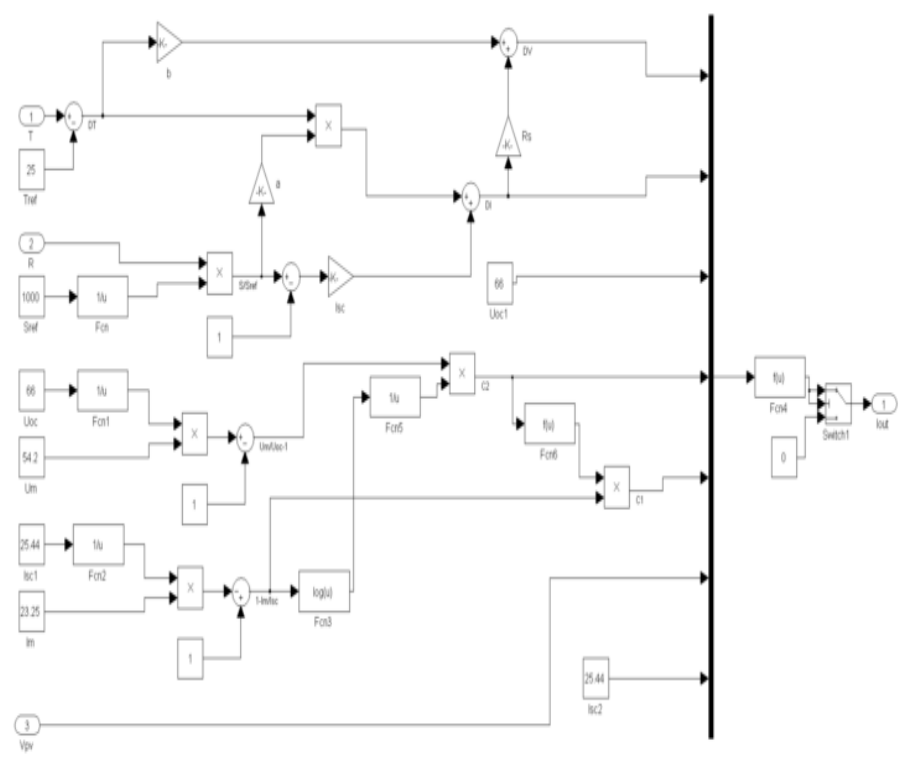

Figure 4. Solar cell simulation model the internal structure

Maximum power point tracking model

In MATLAB, we write MPPT control algorithm by S function, use of the solar cell model above to establish MPPT simulation in MATLAB environment. Simulation schematic is shown in Figure 5.

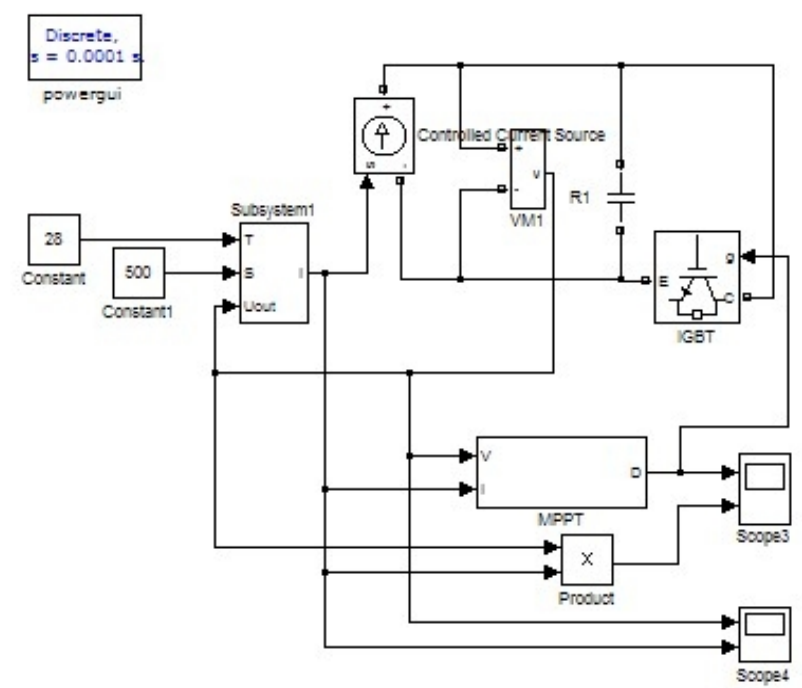

Figure 5. MATLAB simulation diagram of MPPT

The simulation results

In the MPPT of S function, respectively calculate the results according to fixed step length disturbance observation process and step by step length disturbance observation based on the slope of P $\mathrm{V}$ curve. The simulation results are shown in figures 6 and 7. 


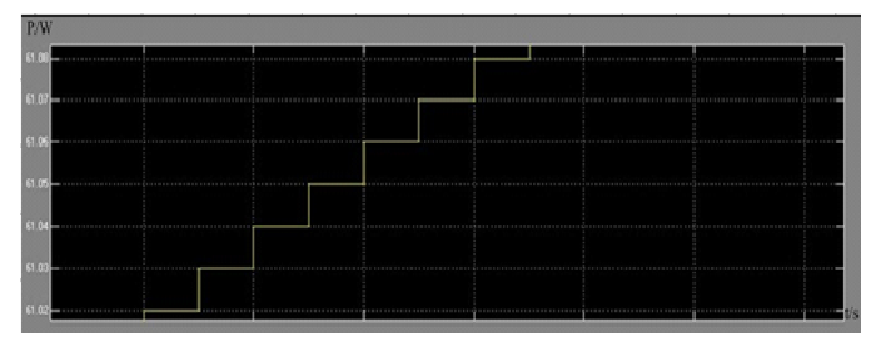

Figure 6 Fixed step disturbance observation.

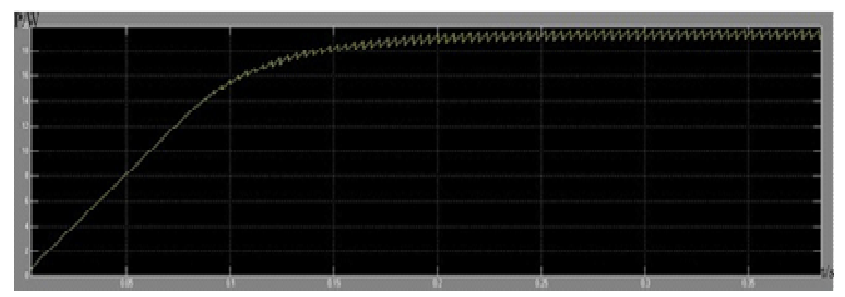

Figure 7 Based P-V curve slope fractional step perturbation and observation method.

As can be seen from Figures 6 and 7, based on the P-V curve slope fractional-step perturbation observation method, dynamic and static resistance are significantly better than fixed step disturbance observation.

\section{REFERENCE}

[1] DAI Xin-ping, MA Guang, YANG Xiao-hong.A Control Method For Maximum Power Tracing Of Photovoltaic Generation System[J].Proceedings of the CSEE,2005,(8):95-99.

[2] Wallzener,M.G,Alves,R.N.C. Current control loop for tracking of maximum power Point supplied for Photovoltaic array[J].Instrumentation and Meastmmaent.IEEE Transactions on Energy Conversion.2004,53(4):1304-1310.

[3] XU Peng-wei, LIU Fei, LIU Bang-yin, DUAN Shan-xu.Analysis, Comparison and Improvement of Several MPPT Methods for PV System[J].Power Electronics,2007,41(5):3-5.

[4] Cui Yan, Cai Binghuang,Li Dayong,Hu Hongxun, Dong Jingwei.Comparative Studies On The Mppt Control Algorithms Of Solar Energy Photovoltaic System[J].Acta Energiae Solaris Sinica,2006,27(6):53-539.

[5] Ye Manyuan.Em ulation ofM PPT Sing le- Stage Photovolta ic Para llelM ains Inverter by Conductance Increm entM ethod[J].Explosion - Proof Electr Ic Mach Ine,2006,41(2):32-35. 